\title{
Polymersomes, smaller than you think: ferrocene as a TEM probe to determine core structure
}

\author{
A. H. Johnston ${ }^{1}$, P. D. Dalton ${ }^{2}$ \& T. A. Newman ${ }^{3}$ \\ ${ }^{1}$ School of Biological Sciences, Biomedical Sciences Building, Bassett Crescent East, University of \\ Southampton, SO16 7PX, United Kingdom \\ ${ }^{2}$ Institute of Health and Biomedical Innovation, Queensland University of Technology, 60 Musk Ave, \\ Kelvin Grove, Australia. \\ ${ }^{3}$ School of Medicine, Building 62, University of Southampton, SO16 7PX, United Kingdom \\ AlexJohnston:ahj@soton.ac.uk
}

\begin{abstract}
By incorporating ferrocene into the hydrophobic membrane of PEG- $b$-PCL polymersome nanoparticles it is possible to selectively visualize their core using Transmission Electron Microscopy (TEM). Two different sizes of ferrocene-loaded polymersomes with mean hydrodynamic diameters of approximately 40 and $90 \mathrm{~nm}$ were prepared. Image analysis of TEM pictures of these polymersomes found that the mean diameter of the core was 4-5 times smaller than the mean hydrodynamic diameter. The values obtained also allow the surface diameter and internal volume of the core to be calculated.
\end{abstract}

KEYWORDS: nanoparticle, characterization, pharmacokinetics, polymersomes, PEG, PCL, TEM. Running title; polymersome core size.

\section{Introduction}

Amphiphilic block copolymers have been shown to self assemble into polymersome nanoparticles in an aqueous environment(Letchford and Burt, 2007). Polymersomes are a class of vesicle, nano-sized spheres which encapsulate an aqueous solution. They have potential to act as targeted drug delivery vehicles, they can carry a payload of therapeutic agents and the surface can be modified with tissue specific targeting moieties. The amphiphilic block copolymers from which they are formed consist of two different polymer chain units joined together, a hydrophilic unit and a hydrophobic unit. Preparation is by slow addition of a block-copolymer solution in a water miscible organic solvent to water. Upon addition the polymer chains self-assemble, the hydrophobic units of the polymer form a 
spherical membrane with an aqueous core and the hydrophilic chains form a surrounding corona as well as line the interior aqueous cavity of the polymersome, Figure 1. Structurally polymersomes are similar to liposomes, however they have several advantages over liposomes which give them greater potential in their use as drug delivery vehicles. The membrane thickness can be tuned by varying the molecular weight of the hydrophobic block copolymer, compared to liposomes this membrane is usually thicker and stronger which gives them greater stability over conventional liposomes(Discher, et al., 1999). Small molecular weight hydrophobic molecules can be incorporated into the membrane(Ghoroghchian, et al., 2006b) and hydrophilic molecules encapsulated within the aqueous core(Lomas, et al., 2007). The polymersome can be surface-functionalized with groups that promote cellular uptake(Christian, et al., 2007; Roy, et al., 2010) or target specific cell types(Park, et al., 2007). There are numerous different block copolymers that can be used to form polymersomes, one of which is poly( $\varepsilon$-caprolactone)- $b$-poly(ethylene glycol)(Ghoroghchian, et al., 2006a; Meng, et al., 2003) (PEG- $b$-PCL). PCL forms the hydrophobic core membrane of the polymersome, it is a FDA approved biodegradable polyester polymer and is currently used in medical applications including suture material. PEG comprises the hydrophilic corona of the polymersome and is noted for its biocompatibility and resistance to both protein adsorption and cell adhesion(Halperin, 1999), resulting in prolongation of circulation times of the polymersome within the body. Characterization of polymersome size in the literature is usually limited to light scattering techniques or negatively stained Transmission Electron Microscopy (TEM) images(Gaumet, et al., 2008), these give little or no information about the size of the polymersome core. The surface area and volume of the core are important factors governing the loading and subsequent release of any incorporated therapeutic agent(Zhou and Feijen, 2008) and is therefore an important physical aspect of the polymersome to characterize. By incorporating the hydrophobic iron (II) compound ferrocene into the polymersome membrane we have been able to image the polymersome core selectively using TEM. Subsequent image analysis enables measurement of the core diameter from which the surface area and volume of the core can be calculated. 


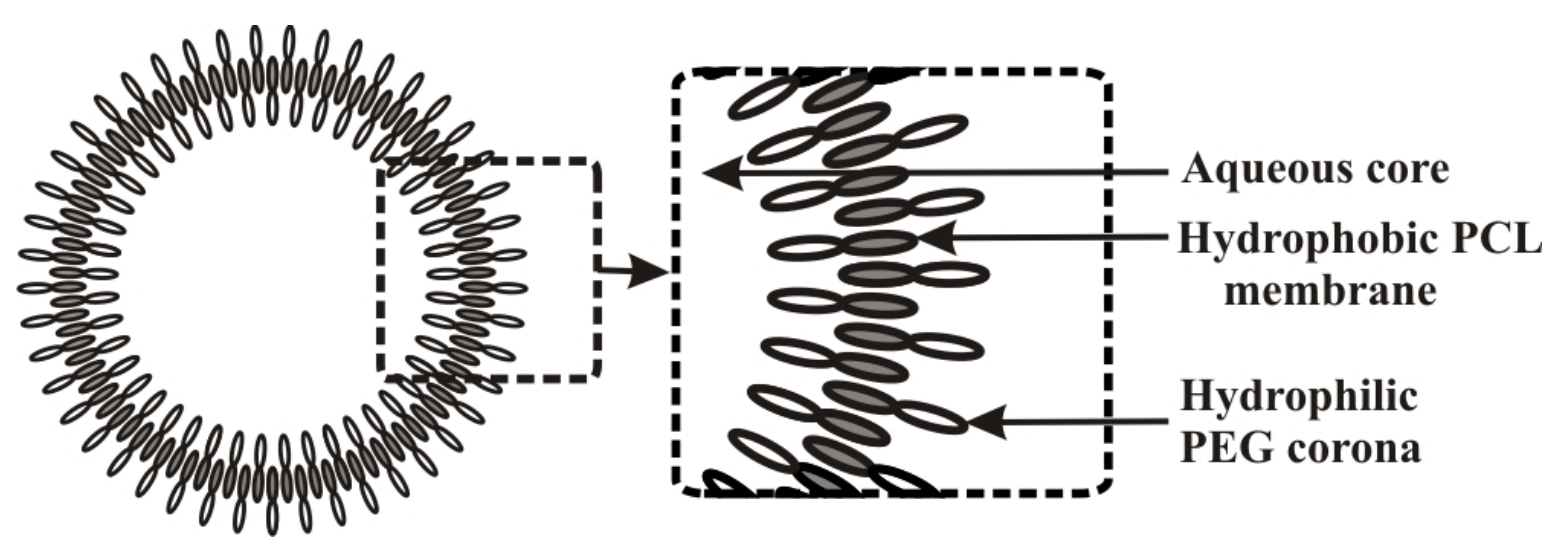

Figure 1. PEG- $b$-PCL block copolymers self assemble in an aqueous environment to form polymersome structures. The PCL units of the polymer form a hydrophobic core membrane, The PEG units form a hydrophilic corona and line the interior cavity of the polymersome.

\section{Experimental Section}

Poly(ethylene glycol)-block-poly( $\varepsilon$-caprolactone) methyl ether (PEG5K-b-PCL5K) and ferrocene supplied from Sigma Aldrich, UK. PEG2K-b-PCL2.6K supplied from Polymer Source Inc, Canada. Dialysis tubing 12-14 kDa cut-off, $6.3 \mathrm{~mm}$ diameter supplied from Medicell International $\mathrm{Ltd}$. $\mathrm{H}_{2} \mathrm{O}$ used was purified using a MilliQ Ultrapure Water Purification System (resistance of $18.2 \mathrm{M} \Omega . \mathrm{cm}$ at 25 $\left.{ }^{\circ} \mathrm{C}\right)$.

For the preparation of unloaded polymersomes, PEG- $b$-PCL block copolymer $(6.0 \mathrm{mg})$ was dissolved in DMF $(0.40 \mathrm{~mL})$ and the solution was placed into a $60 \mathrm{~Hz}$ ultrasonic bath until complete dissolution had occurred. The polymer solution was then added dropwise ( $\sim 1$ drop every 8 seconds) to rapidly stirring MilliQ water $(1.60 \mathrm{~mL})$. The sample was dialysed against MilliQ water for 48 hours with regular changes of water. Analysis by DLS showed the typical size of unloaded PEG2K- $b$-PCL2.6K and PEG5K- $b$-PCL5K polymersomes to be 34.5 (+/-) $4.2 \mathrm{~nm}$ and 82.8 (+/-) $9.8 \mathrm{~nm}$ respectively. The same procedure was used to prepare ferrocene-loaded polymersomes, with the addition that ferrocene was dissolved into the initial DMF-polymer solution at a concentration of $1 \mathrm{mg} / \mathrm{mL}$. Analysis by DLS showed the typical size of ferrocene-loaded PEG2K- $b$-PCL2.6K and PEG5K- $b$-PCL5K polymersomes to be 38.6 (+/-) $5.6 \mathrm{~nm}$ and 90.5 (+/-) $18.2 \mathrm{~nm}$ respectively, Figure 2. Once the samples were prepared, TEM imaging of the unloaded and ferrocene-loaded polymersomes was performed on the same day. DLS analysis over a period of two weeks shows no significant change in hydrodynamic diameter or nanoparticle aggregation. The loading concentration of ferrocene within the polymersomes can be determined by UV absorbance. Briefly, after removal from dialysis $1.1 \mathrm{~mL}$ of polymersome solution is centrifuged at $1500 \mathrm{~g}$ for 10 minutes to separate the ferrocene precipitates. $1 \mathrm{~mL}$ of the supernatant is 
lyophilized, re-dissolved in chloroform and filtered through a $0.2 \mu \mathrm{m}$ PTFE filter. Comparison of the absorbance at $430 \mathrm{~nm}$ with unloaded polymersomes as well as stock solutions of ferrocene allows the loading concentration to be calculated. For PEG2K- $b$-PCL2.6K and PEG5K- $b$-PCL5K polymersomes this was found to be $70.4 \mathrm{nmol} / \mathrm{mL}$ and $74.4 \mathrm{nmol} / \mathrm{mL}$ respectively.
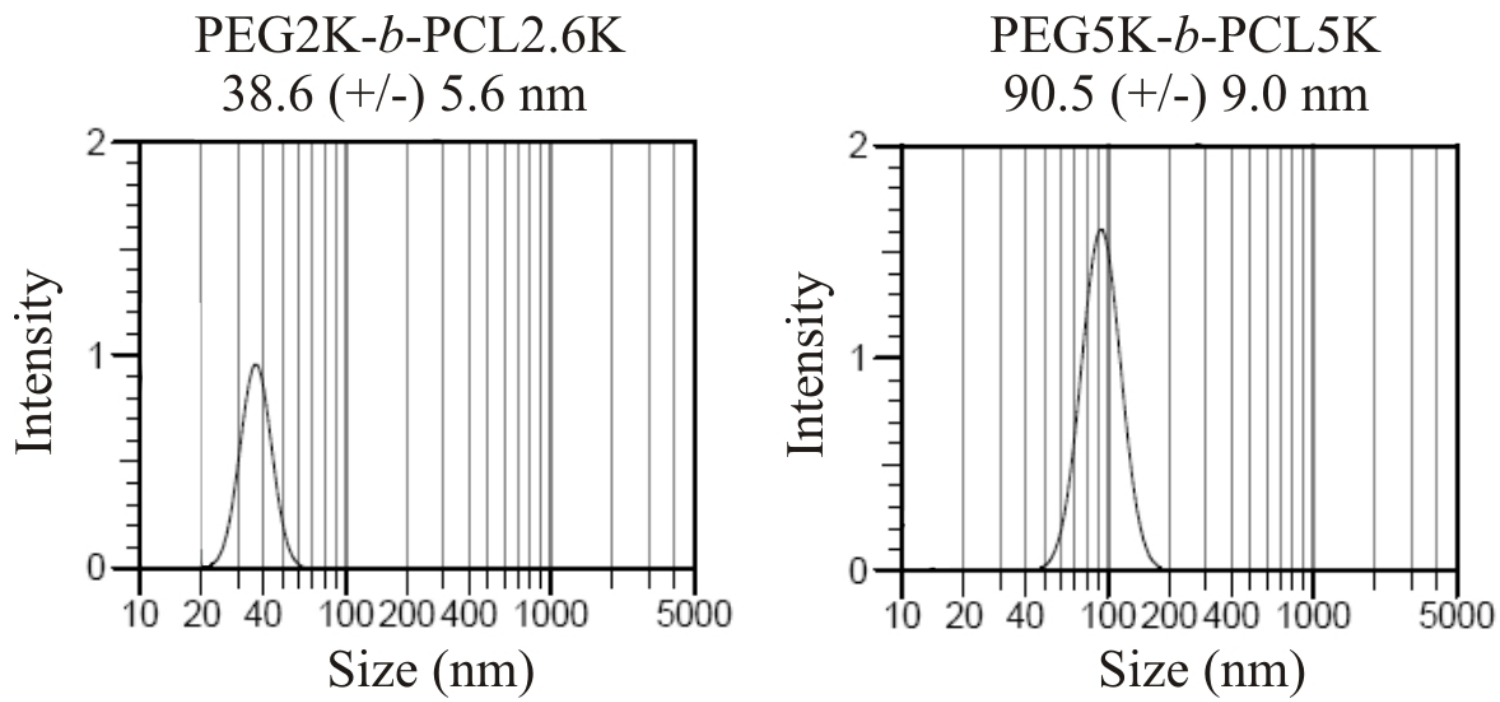

Figure 2. Dynamic light scattering (DLS) results showing the hydrodynamic diameter of the ferrocene-loaded polymersomes.

Hydrodynamic diameter was determined using Dynamic Light Scattering (DLS), $100 \mu \mathrm{L}$ of sample was diluted with $4 \mathrm{~mL}$ of MilliQ water. The solution was filtered into a cuvette through a $0.45 \mu \mathrm{m}$ cellulose acetate membrane. The sample was measured at room temperature, the light was detected at $90^{\circ}$. Data was collected for 150 seconds and repeated 6 times, the size reported is the mean average value and standard deviation. The sample was run on a Coulter N4 Plus particle sizer using N4 Plus Version 1.10 software for data analysis. The polymersomes were also characterized using Transmission Electron Microscopy (TEM). For unloaded polymersomes the sample was first diluted to 1:50 in MilliQ water then filtered through a $0.45 \mu \mathrm{L}$ cellulose acetate membrane filter. $1 \mu \mathrm{L}$ of sample was deposited onto a copper grid coated with formvar and carbon and allowed to air dry. $1 \%$ Uranyl acetate was used as a negative stain, $5 \mu \mathrm{L}$ was applied to the grid, left for 5 seconds, blotted off and left to air dry. For nanoparticles loaded with ferrocene the sample was centrifuged at $1500 \mathrm{~g}$ for 10 minutes, filtered through a $0.45 \mu \mathrm{L}$ cellulose acetate membrane filter and $1 \mu \mathrm{L}$ of sample was deposited onto a copper grid coated with formvar and carbon and allowed to air dry. Determination of polymersome diameter was performed using ImageJ analysis software, version 1.42q(Collins, 2007). The size reported is the mean average value and size deviation. 


\section{Results and Discussion}

Due to their size it is not possible to visualize individual nanoparticles using conventional light microscopy, electron microscopy techniques need to be employed. Transmission Electron Microscopy (TEM) is commonly employed to visualize nanoparticle morphology. Nanoparticles made from dense materials such as quantum dots, silica or gold or iron core nanoparticles are easily identifiable using TEM as spherical structures. However, nanoparticles formed from self assembling polymers such as PEG- $b$-PCL polymersomes do not have a sufficiently dense structure to be readily detected by TEM.

For these a negative stain such as ammonium molybdanate or uranyl acetate is employed. A nanoparticle sample is deposited onto a grid and negatively stained, however the stain only enables the outline of the polymersome to be viewed rather than the polymersome itself, Figure 3 . To obtain more information about polymersome structure we encapsulated ferrocene, a hydrophobic, electron rich Fe(II) compound inside the membrane. The PCL membrane core of the polymersome was imaged selectively and the PEG corona segment is not apparent, Figure 3.
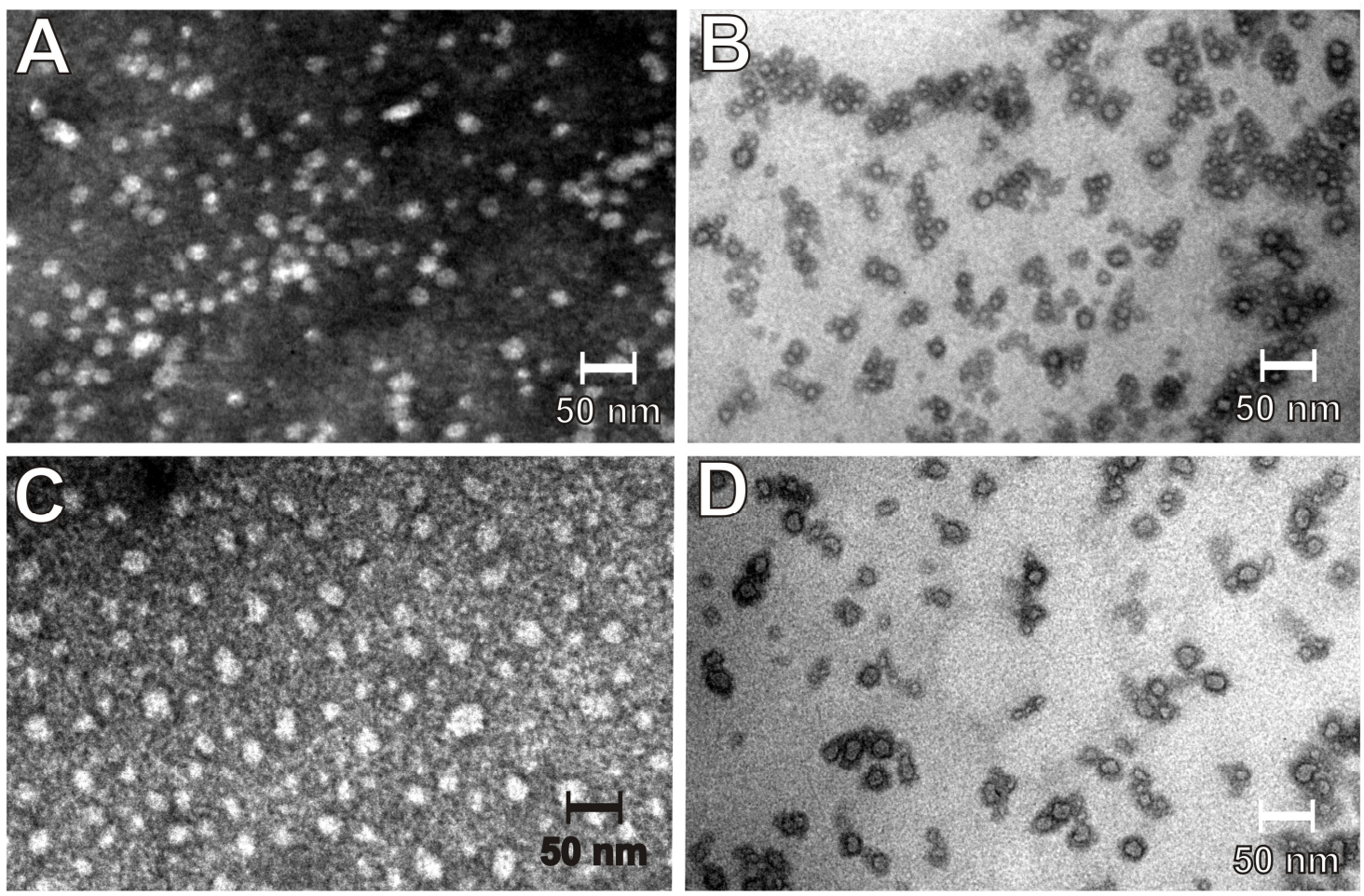

Figure 3. Pictures taken at $100 \times 10^{3}$ magnification. (A) Unloaded PEG2K- $b$-PCL2.6K polymersomes, negatively stained with uranyl acetate. (B) Ferrocene-loaded PEG2K- $b$-PCL2.6K polymersomes. (C) Unloaded PEG5K- $b$-PCL5K polymersomes negatively stained with uranyl acetate. (D) Ferrocene-loaded PEG5K- $b$-PCL5K polymersomes. 
Two different sizes of ferrocene-loaded polymersomes were prepared, each from a different length PEG- $b$-PCL polymer, PEG2K- $b$-PCL2.6K and PEG5K- $b$-PCL5K. The polymersomes were characterized using DLS to measure the hydrodynamic diameter and by analysis of TEM images to give a mean diameter of the polymersome core, Table 1 . The hydrodynamic diameters of polymersomes formed from PEG2K- $b$-PCL2.6K and PEG5K-b-PCL5K were 38.6 (+/-) $5.6 \mathrm{~nm}$ and 90.5 (+/-) $9.0 \mathrm{~nm}$ respectively, the mean core diameter and standard deviation measured by TEM are 9.5 (+/-) $2.8 \mathrm{~nm}$ and $18.0(+/-) 3.6 \mathrm{~nm}$. The difference in size between the polymersomes formed from the two polymers is due to the ratio of the hydrophobic and hydrophilic chains. For PEG2K- $b$ PCL2.6K and PEG5K- $b$-PCL5K this is 1:1.3 and 1:1 respectively. With polymersomes formed from PEG2K- $b$-PCL2.6K the hydrodynamic diameter of the nanoparticles is approximately 4 times greater than the mean diameter of the core determined using TEM and 5 times with PEG5K- $b$-PCL5K. The mean core diameter for polymersomes formed from PEG5K- $b$-PCL5K is also approximately double that for those formed from PEG2K-b-PCL2.6K. Halving the mean core diameter to give the radius allows values for the surface area of the hydrophobic membrane as well as volume of the internal cavity to be calculated. The membrane surface area of polymersomes formed from PEG2K- $b$ PCL2.6K and PEG5K- $b$-PCL5K were $305.6 \mathrm{~nm}^{2}$ and $1056.0 \mathrm{~nm}^{2}$ respectively, and the volume of the cores $565.7 \mathrm{~nm}^{3}$ and $3416.0 \mathrm{~nm}^{3}$. It can be seen that the increase in hydrophobic membrane surface area between polymersomes formed from PEG5K- $b$-PCL5K and PEG2K- $b$-PCL2.6K is approximately 3.5 fold and the increase in core volume 6 fold.

\begin{tabular}{c|ccccc}
\hline Nanoparticle matrix & $\begin{array}{c}\text { Hydrodynamic } \\
\text { diameter }(\mathrm{nm})\end{array}$ & $\begin{array}{c}\text { Mean core } \\
\text { diameter } \\
\text { by TEM (nm) }\end{array}$ & $\begin{array}{c}\text { Mean core } \\
\text { radius } \\
\text { by TEM (nm) }\end{array}$ & $\begin{array}{c}\text { Mean core } \\
\text { surface } \\
\text { area }\left(\mathrm{nm}^{2}\right)\end{array}$ & $\begin{array}{c}\text { Mean core } \\
\text { volume } \\
\left(\mathrm{nm}^{3}\right)\end{array}$ \\
\hline PEG2K- $b$-PCL2.6K & $38.6(+/-) 5.6$ & $9.5(+/-) 2.8$ & $4.7(+/-) 1.4$ & 305.6 & 565.7 \\
\hline PEG5K- $b$-PCL5K & $90.5(+/-) 9.0$ & $18.0(+/-) 3.6$ & $9.0(+/-) 1.8$ & 1056.0 & 3416.0
\end{tabular}

Table 1. The hydrodynamic diameter obtained by light scattering and the mean core diameter, surface area and volume obtained by TEM. The standard deviation is given as the size distribution.

It has previously been shown that nanoparticle size has a large influence on their biodistribution(Banerjee, et al., 2002; Moghimi, 1995a; b) as well as their cellular uptake (Chithrani, et al., 2009; Gaumet, et al., 2009) and is therefore an important factor to consider in their design as drug delivery vehicles. However, as Gaumet et al highlight(Gaumet, et al., 2008), the characterization of 
nanoparticle size in the literature is usually very limited with usually often one and in some cases two techniques employed. This is especially true of polymeric nanoparticles where size is most commonly determined either by analysis of negatively stained TEM images or by dynamic light scattering. Negatively stained TEM images are usually unclear and whilst it is possible to ascertain spherical morphology, it can be difficult to calculate size. Light scattering techniques measure hydrodynamic diameter, this measurement is usually made in water or buffer solution and is not representative of the biological medium the particles will meet after administration. As we have shown, due to the PEG corona, the hydrodynamic diameter measured using light scattering can be 4-5 times larger than the diameter of the polymersome core. As one of the factors which controls nanoparticle uptake is the level of protein adsorption(Chithrani, et al., 2009), this is more dependant on the surface area of the polymersome core than the hydrodynamic diameter that also includes the PEG corona. Accurate determination of core diameter is also important in considering the drug delivery of the polymersome, as rate of payload release is dependant on the surface area and volume of the core(Zhou and Feijen, 2008). The use of ferrocene as a TEM probe reported here allows a more accurate determination of the core diameter of polymeric nanoparticles, it could be used in conjunction with light scattering and other techniques to improve polymersome characterization.

\section{Acknowledgements}

This work was supported by European Community; Grant number: NMP4-CT-2006-026556 Integrated EU project.

\section{References}

Banerjee, T., Mitra, S., Singh, A.K., Sharma, R.K. and Maitra, A., 2002. Preparation, characterization and biodistribution of ultrafine chitosan nanoparticles. International Journal of Pharmaceutics, 243 (1-2), 93-105.

Chithrani, B.D., Stewart, J., Allen, C. and Jaffray, D.A., 2009. Intracellular uptake, transport, and processing of nanostructures in cancer cells. Nanomedicine: Nanotechnology, Biology and Medicine, 5 (2), 118-127.

Christian, N.A., Milone, M.C., Ranka, S.S., Li, G.Z., Frail, P.R., Davis, K.P., Bates, F.S., Therien, M.J., Ghoroghchian, P.P., June, C.H. and Hammer, D.A., 2007. Tatfunctionalized near-infrared emissive polymersomes for dendritic cell labeling. Bioconjugate Chemistry, 18 (1), 31-40.

Collins, T.J., 2007. ImageJ for microscopy. Biotechniques, 43 (1), 25-30.

Discher, B.M., Won, Y.Y., Ege, D.S., Lee, J.C.M., Bates, F.S., Discher, D.E. and 
Hammer, D.A., 1999. Polymersomes: Tough vesicles made from diblock copolymers. Science, 284 (5417), 1143-1146.

Gaumet, M., Gurny, R. and Delie, F., 2009. Localization and quantification of biodegradable particles in an intestinal cell model: The influence of particle size. European Journal of Pharmaceutical Sciences, 36 (4-5), 465-473.

Gaumet, M., Vargas, A., Gurny, R. and Delie, F., 2008. Nanoparticles for drug delivery: The need for precision in reporting particle size parameters. European Journal of Pharmaceutics and Biopharmaceutics, 69 (1), 1-9.

Ghoroghchian, P.P., Li, G.Z., Levine, D.H., Davis, K.P., Bates, F.S., Hammer, D.A. and Therien, M.J., 2006a. Bioresorbable vesicles formed through spontaneous selfassembly of amphiphilic poly(ethylene oxide)-block-polycaprolactone.

Macromolecules, 39 (5), 1673-1675.

Ghoroghchian, P.P., Lin, J.J., Brannan, A.K., Frail, P.R., Bates, F.S., Therien, M.J. and Hammer, D.A., 2006b. Quantitative membrane loading of polymer vesicles. Soft Matter, 2 (11), 973-980.

Halperin, A., 1999. Polymer brushes that resist adsorption of model proteins: Design parameters. Langmuir, 15 (7), 2525-2533.

Letchford, K. and Burt, H., 2007. A review of the formation and classification of amphiphilic block copolymer nanoparticulate structures: micelles, nanospheres, nanocapsules and polymersomes. European Journal of Pharmaceutics and Biopharmaceutics, 65 (3), 259-269.

Lomas, H., Canton, I., MacNeil, S., Du, J., Armes, S.P., Ryan, A.J., Lewis, A.L. and Battaglia, G., 2007. Biomimetic $\mathrm{pH}$ sensitive polymersomes for efficient DNA encapsulation and delivery. Advanced Materials, 19 (23), 4238-4243.

Meng, F.H., Hiemstra, C., Engbers, G.H.M. and Feijen, J., 2003. Biodegradable polymersomes. Macromolecules, 36 (9), 3004-3006.

Moghimi, S.M., 1995a. Exploiting Bone-Marrow Microvascular Structure for DrugDelivery and Future Therapies. Advanced Drug Delivery Reviews, 17 (1), 61-73.

Moghimi, S.M., 1995b. Mechanisms of Splenic Clearance of Blood-Cells and Particles - Towards Development of New Splenotropic Agents. Advanced Drug Delivery Reviews, 17 (1), 103-115.

Park, I.K., Lasiene, J., Chou, S.H., Horner, P.J. and Pun, S.H., 2007. Neuron-specific delivery of nucleic acids mediated by Tet(1)-modified poly(ethylenimine). Journal of Gene Medicine, 9 (8), 691-702.

Roy, S., Johnston, A.H., Newman, T.A., Glueckert, R., Dudas, J., Bitsche, M., Corbacella, E., Rieger, G., Martini, A. and Schrott Fischer, A., 2010. Cell-specific targeting in the mouse inner ear using nanoparticles conjugated with a neurotrophin derived peptide ligand: Potential tool for drug delivery. International Journal of Pharmaceutics, in press. DOI 10.1016/j.ijpharm.2010.02.003 
Zhou, W. and Feijen, J., 2008. Biodegradable polymersomes for controlled drug release. Journal of Controlled Release, 132 (3), e35-e36. 